\title{
Bound state solutions of the Schrödinger equation with energy- dependent molecular Kratzer potential via asymptotic iteration method
}

Akpan Ndem Ikot ${ }^{1}{ }^{\oplus}$, Uduakobong Okorie ${ }^{1,2}{ }^{\oplus}$, Alalibo Thompson Ngiangia ${ }^{1}$,Clement Atachegbe Onate ${ }^{3}$, Collins Okon Edet $^{1+}{ }^{+}$, Ita Okon Akpan ${ }^{4}$, Precious Ogbonda Amadi ${ }^{1}$

1. University of Port Harcourt, Department of Physics, Theoretical Physics Group, Nigeria

2. Akwa Ibom State University, Department of Physics, Ikot Akpaden, Nigeria

3. Landmark University, Department of Physical Sciences, Omu-Aran, Nigeria

4. University of Calabar, Department of Physics, Nigeria

+Corresponding author: Collins Okon Edet, email address: collinsokonedet@gmail.com

\section{ARTICLE INFO}

\section{Article history:}

Received: July 21, 2019

Accepted: October 24, 2019

Published: January 1, 2020

\section{Keywords:}

1. Kratzer potential

2. Schrödinger equation

3. asymptotic iteration method

4. bound state
ABSTRACT: In this paper, we obtained the exact bound state energy spectrum of the Schrödinger equation with energy dependent molecular Kratzer potential using asymptotic iteration method (AIM). The corresponding wave function expressed in terms of the confluent hypergeometric function was also obtained. As a special case, when the energy slope parameter in the energydependent molecular Kratzer potential is set to zero, then the well-known molecular Kratzer potential is recovered. Numerical results for the energy eigenvlaues are also obtained for different quantum states, in the presence and absence of the energy slope parameter. These results are discussed extensively using graphical representation. Our results are seen to agree with the results in literature.

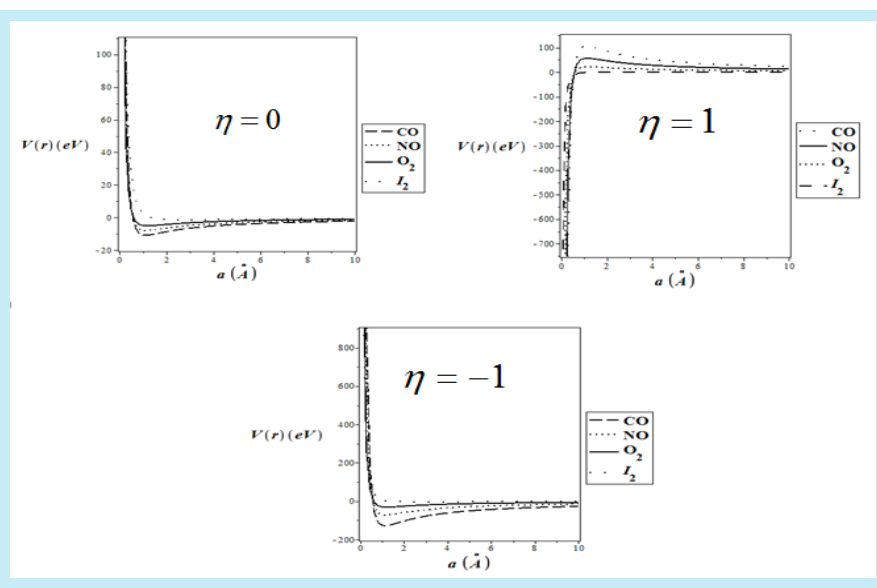

\section{Introduction}

The exact or approximate solutions of the Schrödinger equations play a vital role in many branches of modern physics and chemistry ${ }^{1,2}$. The solution of this equation is used in the description of particle dynamics in the non-relativistic regime $^{3,4}$. Even though the Schrödinger equation was developed many decades ago, it is still very challenging to solve it analytically $y^{5,6}$. The solution of the Schrödinger equation contains all the necessary information needed for the full description of a quantum state such as the probability density and entropy of the system ${ }^{7,8}$. The Schrödinger equation with many physical potentials model have been investigated in recent times with different advance mathematical technique such as Nikiforov-Uvarov (NU) method $^{9-11}$, asymptotic iteration method (AIM) ${ }^{12-16}$, functional analysis approach ${ }^{16}$, supersymmetric quantum mechanics (SUSYQM) ${ }^{17-20}$ among others $^{21}$. One of such potential models is the Kratzer potential ${ }^{22}$,

$$
V(r)=-2 D\left(\frac{a}{r}-\frac{1}{2} \frac{a^{2}}{r^{2}}\right)
$$

where $D$ is the dissociation energy and $a$ is the equilibrium internuclear length.

The Kratzer potential has been used as a potential model to describe internuclear vibration of diatomic molecules ${ }^{23,24}$. Many authors have investigated the bound state solutions of the Kratzer potential within relativistic and nonrelativistic quantum mechanics ${ }^{25,26}$. Recently, Budaca $^{27}$ studied an energy-dependent Coulomb- 
like potential within the framework of Bohr Hamiltonian. Furthermore, Budaca ${ }^{27}$ had reported that the energy dependence on the coupling constant of the potential drastically changes the analytical properties of wave function and the corresponding eigenvalues of the system. The energy-dependent potentials have been studied in nuclear physics with applications to quark confinement $^{28,29}$. Several researchers have also given great attention to investigate the energy dependent potentials ${ }^{30,31}$. Boumali and Labidi ${ }^{32}$ solved the Klein-Gordon equation with an energydependent potential, the Shannon and Fisher information theory was also considered. Also, Lombard et.al. ${ }^{33}$ studied the wave equation energydependent potential for confined systems. Therefore, the energy dependent potential in the Schrödinger equation or other wave equation in physics has many applications such as features in spectrum of confined systems and heavy quark systems in nuclear and molecular physics ${ }^{34}$.

In this paper, we shall study the influence of the energy-dependent Kratzer potential on some diatomic molecules defined as:

$V(r, E)=-2 D\left(\frac{a(1+\eta E)}{r}-\frac{1}{2} \frac{a^{2}(1+\eta E)}{r^{2}}\right)$

where the energy slope parameter $\eta$ must be positive definite in order to describe a physical system $^{27}$.

The shape of the energy dependent Kratzer molecular potential with different energy slope parameters, as it varies with equilibrium internuclear length are illustrated in Fig. 1A-1C, for four selected diatomic molecules $\left(\mathrm{CO}, \mathrm{NO}, \mathrm{O}_{2}\right.$ and $\mathrm{I}_{2}$ ).

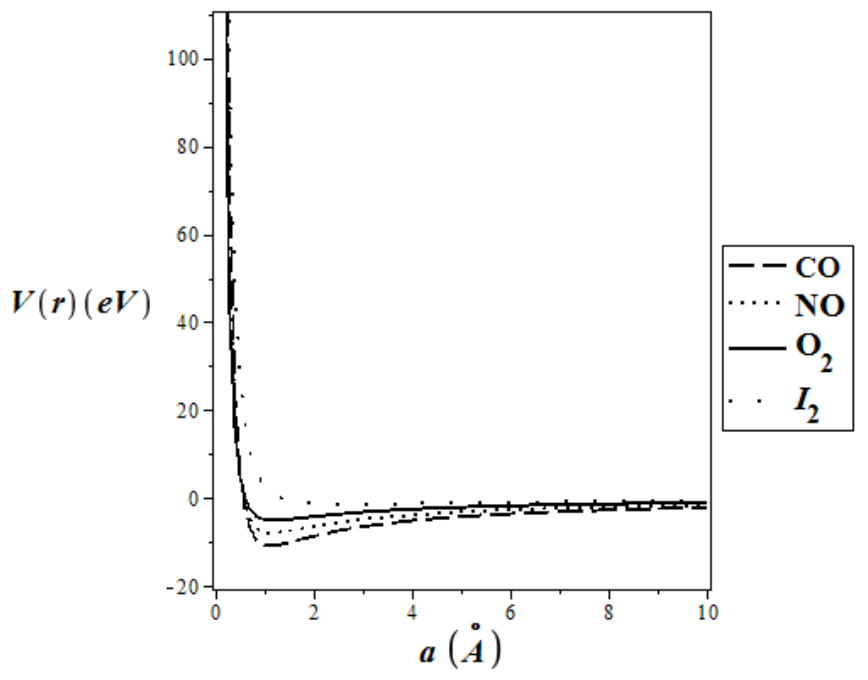

Figure 1A. The shape of Energy-dependent Kratzer molecular potential for different diatomic molecules, with $\eta=0$.

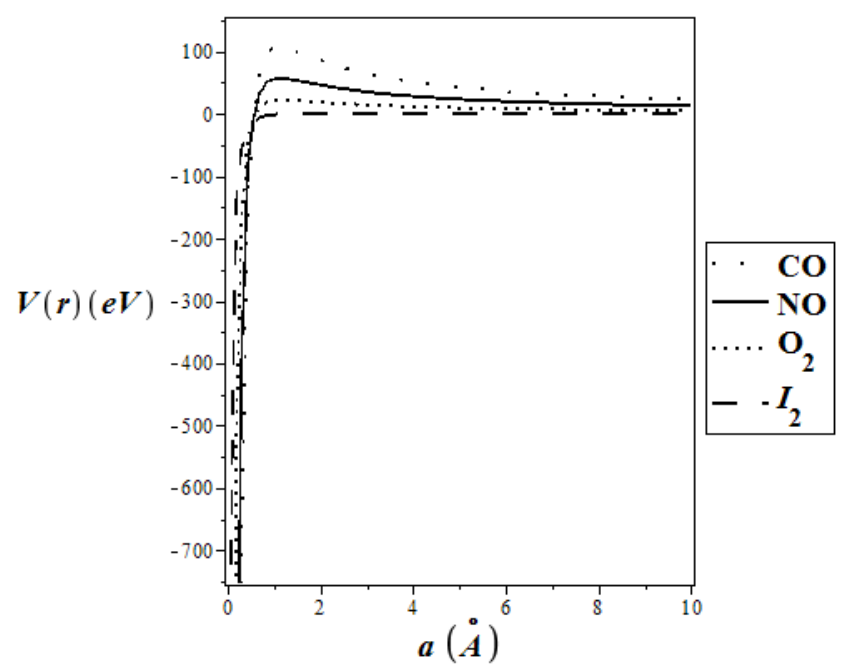

Figure 1B. The shape of Energy-dependent Kratzer Molecular Potential for different diatomic molecules, with $\eta=1$. 


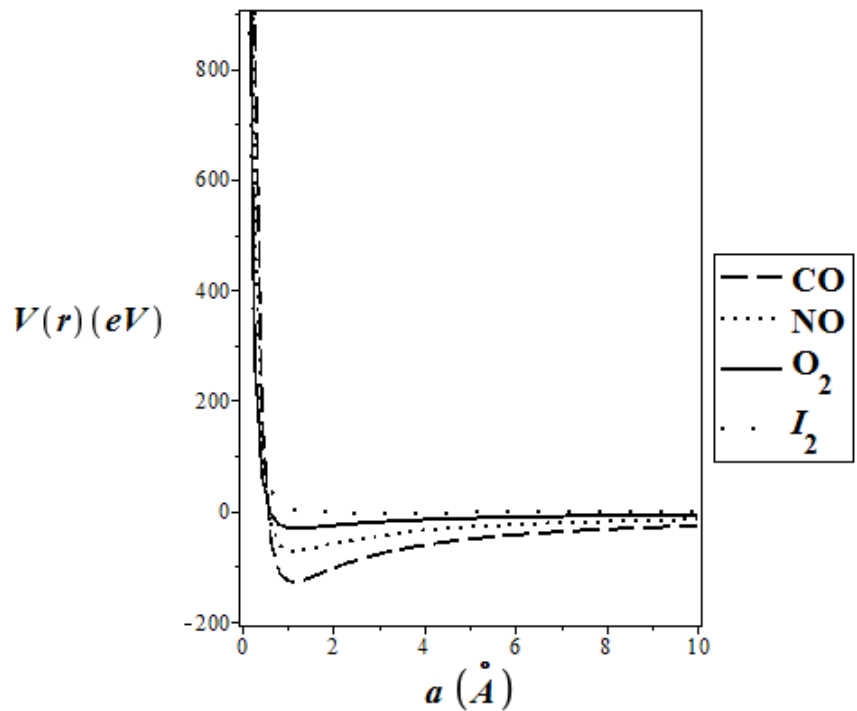

Figure 1C. The shape of Energy-dependent Kratzer molecular potential for different diatomic molecules, with $\eta=-1$.

\section{Asymptotic Iteration Method}

The AIM has been proposed and used to solve the homogenous linear second-order differential equation of the form ${ }^{12-16}$ :

$$
y^{\prime \prime}(x)=\lambda_{0}(x) y^{\prime}(x)+s_{0}(x) y(x)
$$

where $\lambda_{0} \neq 0$ and the prime denote the derivative with respect to $x$.

The functions, $s_{0}(x)$ and $\lambda_{0}(x)$ must be sufficiently differentiable. Differentiating Eq. 3 with respect to $x$, we get

$$
y^{\prime \prime \prime}(x)=\lambda_{1}(x) y^{\prime}(x)+s_{1}(x) y(x)
$$

where,

$$
\begin{aligned}
& \lambda_{1}(x)=\lambda_{0}^{\prime}(x)+\lambda_{0}^{2}(x)+s_{0}(x) \\
& s_{1}(x)=s_{0}^{\prime}(x)+s_{0}(x) \lambda_{0}(x)
\end{aligned}
$$

Taking the second derivative of Eq. 3 yields

$$
y^{\prime \prime \prime \prime}(x)=\lambda_{2}(x) y^{\prime}(x)+s_{2}(x) y(x)
$$

$$
\begin{aligned}
& \lambda_{2}(x)=\lambda_{1}^{\prime}(x)+\lambda_{0}(x) \lambda_{1}(x)+s_{1}(x) \\
& s_{2}(x)=s_{1}^{\prime}(x)+s_{0}(x) \lambda_{1}(x)
\end{aligned}
$$

Again by taking the $(k+1)$ th and $(k+2)$ th order derivative of Eq. 3 for $k=1,2,3 \ldots$, we obtain the following differential equations:

$$
\begin{aligned}
& y^{(k+1)}(x)=\lambda_{k-1}(x) y^{\prime}(x)+s_{k-1}(x) y(x) \\
& y^{(k+2)}(x)=\lambda_{k}(x) y^{\prime}(x)+s_{k}(x) y(x)
\end{aligned}
$$

where,

$$
\begin{aligned}
& \lambda_{k-1}(x)=\lambda_{k-2}^{\prime}(x)+\lambda_{0}(x) \lambda_{k-2}(x)+s_{k-2}(x) \\
& s_{k-1}(x)=s_{0}(x) \lambda_{k-2}(x)+s_{k-2}^{\prime}(x) \\
& \lambda_{k}(x)=\lambda_{k-1}^{\prime}(x)+\lambda_{0}(x) \lambda_{k-1}(x)+s_{k-1}(x) \\
& s_{k}(x)=s_{0}(x) \lambda_{k-1}(x)+s_{k-1}^{\prime}(x)
\end{aligned}
$$

Solving Eq. 8, we obtain the following relation:

$$
\frac{y^{(k+2)}(x)}{y^{(k+1)}(x)}=\frac{\lambda_{k}(x)\left[y^{\prime}(x)+\frac{s_{k}(x)}{\lambda_{k}(x)} y(x)\right]}{\lambda_{k-1}(x)\left[y^{\prime}(x)+\frac{s_{k-1}(x)}{\lambda_{k-1}(x)} y(x)\right]}
$$

For sufficiently large values of $k$, the $\alpha(x)$ values are obtained as

$$
\frac{s_{k}(x)}{\lambda_{k}(x)}=\frac{s_{k-1}(x)}{\lambda_{k-1}(x)}=\alpha(x)
$$

This method consists of converting the Schrödinger-like equation into the form of Eq. 3 for a given potential model. The corresponding energy eigenvalues are calculated by means of the quantization condition $^{12-16}$.

$$
\delta_{k}(x)=s_{k}(x) \lambda_{k-1}(x)-\lambda_{k}(x) s_{k-1}(x), k=1,2,3 \ldots
$$

The general solutions of Eq. 3 is obtain from Eq. 10 as:

where,

$$
y(x)=\exp \left(-\int^{x} \alpha\left(x_{1}\right) d x_{1}\right)\left[C_{2}+C_{1} \int^{x} \exp \left(\int^{x}\left[\lambda_{0}\left(x_{2}\right)+2 \alpha\left(x_{2}\right)\right] d x_{2}\right) d x_{1}\right]
$$

where $C_{1}$ and $C_{2}$ are integration constant. 
Also, the eigenfunction can be obtained by transforming the Schrödinger-like equation of the form:

$$
y^{\prime \prime}(x)=2\left(\frac{a x^{N+1}}{1-b x^{N+2}}-\frac{t+1}{x}\right) y^{\prime}(x)-\frac{W x^{N}}{1-b x^{N+2}} y(x)
$$

The exact solutions for Eq. 14 are given by

$$
y(x)=(-1)^{2} C(N+2)(\sigma)_{n 2} F_{1}\left(-n, \rho+n ; \sigma ; b x^{N+2}\right)
$$

where,

$$
(\sigma)_{n}=\frac{\Gamma(\sigma+n)}{\sigma}, \sigma=\frac{2 t+N+3}{N+2}, \rho=\frac{(2 t+1) b+2 a}{(N+2) b}
$$

\section{Bound state solution of the Schrödinger equation with energy-dependent Kratzer potential}

The radial part of the Schrödinger equation with energy dependent potential $V\left(r, E_{n l}\right)$ reads $^{35}$ :

$\frac{d^{2} \psi_{n l}}{d r^{2}}+\frac{2 \mu}{\hbar^{2}}\left(E_{n l}-V\left(r, E_{n l}\right)-\frac{l(l+1) \hbar^{2}}{2 \mu r^{2}}\right) \psi_{n l}(r)=0$

where $\mu$ is the reduced mass of the molecules, $E_{n l}$ is the energy of the system, $\hbar$ denotes the reduced Planck constant, $n$ and $\ell$ represent the principal and orbital angular momentum quantum numbers respectively.

Substituting Eq. 1 into Eq. 17 yields,

$\frac{d^{2} \psi_{n l}}{d r^{2}}+\frac{2 \mu}{\hbar^{2}}\left(E_{n l}+2 D\left(\frac{a(1+\eta E)}{r}-\frac{1}{2} \frac{a^{2}(1+\eta E)}{r^{2}}\right)-\frac{l(l+1) \hbar^{2}}{2 \mu r^{2}}\right) \psi_{n l}(r)=0$

Now using a new variable transformation, $y=a r$, we obtain a second order differential equation of the form:

$\frac{d^{2} \psi_{n l}}{d y^{2}}+\left[-\varepsilon_{n}^{2}+\frac{\beta}{y}-\frac{\gamma(\gamma+1)}{y^{2}}\right] \psi_{n l}(y)=0$

with the following definitions for the used parameters:

$$
\begin{aligned}
& \varepsilon_{n}^{2}=-\frac{2 \mu E_{n l}}{\hbar^{2} a^{2}}, \beta=\frac{4 \mu D(1+\eta E)}{\hbar^{2}}, \\
& \gamma(\gamma+1)=\frac{2 \mu D a^{2}(1+\eta E)}{\hbar^{2}}+l(l+1)
\end{aligned}
$$

In order to transform Eq. 19 into form suitable for the AIM, we write the wave function in the form:

$$
\psi_{n l}(y)=y^{\gamma+1} e^{-\varepsilon_{n} y} f(y)
$$

Substituting Eq. 21 into Eq. 19, we obtain:

$$
f^{\prime \prime}(y)=\left[\frac{2 \varepsilon_{n} y-2(\gamma+1)}{y}\right] f^{\prime}(y)+\left[\frac{2 \varepsilon_{n}(\gamma+1)-\beta}{y}\right] f(y)
$$

where,

$$
\begin{aligned}
& \lambda_{0}(y)=\frac{2 \varepsilon_{n} y-2(\gamma+1)}{y}, \\
& s_{0}(y)=\frac{2 \varepsilon_{n}(\gamma+1)-\beta}{y}
\end{aligned}
$$

By applying the AIM quantization of Eq. 12 by substituting Eq. 23, we obtain the following iterations:

$$
\begin{aligned}
& \frac{s_{0}}{\lambda_{0}}=\frac{s_{1}}{\lambda_{1}} \Rightarrow \varepsilon_{0}=\frac{\beta}{2\left(\gamma_{0}+1\right)}, n=0, \\
& \frac{s_{1}}{\lambda_{1}}=\frac{s_{2}}{\lambda_{2}} \Rightarrow \varepsilon_{1}=\frac{\beta}{2\left(\gamma_{1}+2\right)}, n=1, \\
& \frac{s_{2}}{\lambda_{2}}=\frac{s_{3}}{\lambda_{3}} \Rightarrow \varepsilon_{2}=\frac{\beta}{2\left(\gamma_{2}+3\right)}, n=2
\end{aligned}
$$

Generally, for arbitrary $n$, we have

$\varepsilon_{n}=\frac{\beta}{2\left(\gamma_{n}+n+1\right)}$

Using Eq. 20 with Eq. 25, the energy eigenvalues of the Schrödinger equation with energy-dependent Kratzer potential is obtained as: 


$$
E_{n l}=-\frac{1}{8 \mu} \frac{\beta^{2} \hbar^{2} a^{2}}{\left[n+\frac{1}{2}+\frac{1}{2} \sqrt{1+4 l(l+1)+\frac{8 \mu D a^{2}\left(1+\eta E_{n}\right)}{\hbar^{2}}}\right]^{2}}
$$

This is a very complicated transcendental energy equation. As a special case, when the energy slope parameter $\eta=0$, Eq. 26 reduces to the result of the standard Kratzer potential given as:

$$
E_{n l}=-\frac{2 \mu D^{2} a^{2}}{\hbar^{2}\left(n+\frac{1}{2}+\sqrt{\left(l+\frac{1}{2}\right)^{2}+\frac{2 \mu D a^{2}}{\hbar^{2}}}\right)^{2}}
$$

This result is consistent with those reported by Bayrak, Boztosun and $\mathrm{Ciftci}^{29}$.

\section{Results and Discussion}

We compute the energy eigenvalues (in $\mathrm{eV}$ ) of energy dependent Kratzer molecular potential for $\mathrm{CO}, \mathrm{NO}, \mathrm{O}_{2}$ and $\mathrm{I}_{2}$ diatomic molecules. This was done using the spectroscopic parameters given in Table 1, and the conversion $\hbar c=1973.29 \mathrm{eV} \AA^{36}$.

Table 1. Spectroscopic parameters of the various diatomic molecules $^{29}$

\begin{tabular}{|c|c|c|c|}
\hline Molecule & $D(\mathrm{eV})$ & $a(\AA)$ & $\mu(\mathrm{amu})$ \\
\hline $\mathrm{CO}$ & 10.84514471 & 1.1282 & 6.860586000 \\
\hline $\mathrm{NO}$ & 8.043782568 & 1.1508 & 7.468441000 \\
\hline $\mathrm{O}_{2}$ & 5.156658828 & 1.2080 & 7.997457504 \\
\hline$I_{2}$ & 1.581791863 & 2.6620 & 63.45223502 \\
\hline
\end{tabular}

The energy eigenvalues of energy dependent molecular Kratzer potential for the ground state diatomic molecules selected are shown in Tables 2 and 3 , in the absence of the energy slope parameter $\eta$.

Table 2. Energy eigenvalues (in $\mathrm{eV}$ ) of energy dependent Kratzer molecular potential for different values of $n$ and $\ell$ for ground states $\mathrm{CO}$ and $\mathrm{NO}$ diatomic molecules, with $\eta=0$.

\begin{tabular}{|l|l|l|l|l|l|}
\hline$n$ & $\ell$ & $C O$ & $\mathrm{CO}^{29}$ & $N O$ & $\mathrm{NO}^{29}$ \\
\hline 0 & 0 & -10.79431511 & -10.79431532 & -8.002659248 & -8.002659417 \\
\hline 1 & 0 & -10.69383928 & -10.69383992 & -7.921456326 & -7.921456839 \\
\hline & 1 & -10.69337058 & -10.69337123 & -7.921043312 & -7.921043834 \\
\hline & & & & & \\
\hline 2 & 0 & -10.59475984 & -10.59476089 & -7.841483114 & -7.841483956 \\
\hline & 1 & -10.59429764 & -10.59429869 & -7.841076336 & -7.841077188 \\
\hline & 2 & -10.59337335 & -10.59337441 & -7.840262914 & -7.840263771 \\
\hline 3 & 0 & -10.49705101 & -10.49705246 & -7.762714900 & -7.762716066 \\
\hline & 1 & -10.49659519 & -10.49659664 & -7.762314236 & -7.762315413 \\
\hline & 2 & -10.49568366 & -10.49568512 & -7.761513040 & -7.761514218 \\
\hline & 3 & -10.49431667 & -10.49431814 & -7.760311552 & -7.760312744 \\
\hline & & & & & \\
\hline 4 & 0 & -10.40068763 & -10.40068947 & -7.685127602 & -7.685129079 \\
\hline & 1 & -10.40023807 & -10.40023992 & -7.684732928 & -7.684734417 \\
\hline & 2 & -10.39933907 & -10.39934092 & -7.683943714 & -7.683945203 \\
\hline & 3 & -10.39799086 & -10.39799272 & -7.682760192 & -7.682761696 \\
\hline & 4 & -10.39619379 & -10.39619567 & -7.681182728 & -7.681184246 \\
\hline & & & & & \\
\hline
\end{tabular}




\begin{tabular}{|l|l|l|l|l|l|}
\hline 5 & 0 & -10.30564512 & -10.30564735 & -7.608697730 & -7.608699509 \\
\hline & 1 & -10.30520171 & -10.30520394 & -7.608308928 & -7.608310719 \\
\hline & 2 & -10.30431500 & -10.30431723 & -7.607531456 & -7.607533248 \\
\hline & 3 & -10.30298523 & -10.30298747 & -7.606365544 & -7.606367349 \\
\hline & 4 & -10.30121273 & -10.30121499 & -7.604811550 & -7.604813368 \\
\hline & 5 & -10.29899796 & -10.29900024 & -7.602869968 & -7.602871795 \\
\hline
\end{tabular}

Table 3. Energy eigenvalues (in eV) of energy dependent Kratzer molecular potential for different values of $n$ and $\ell$ for ground states $\mathrm{O}_{2}$ and $\mathrm{I}_{2}$ diatomic molecules, with $\eta=0$.

\begin{tabular}{|l|l|l|l|l|l|}
\hline$n$ & $\ell$ & $\mathrm{O}_{2}$ & $\mathrm{O}_{2}{ }^{29}$ & $I_{2}$ & $I_{2}{ }^{29}$ \\
\hline 0 & 0 & -5.126358490 & -5.126358625 & -1.579082564 & -1.579082577 \\
\hline 1 & 0 & -5.066640766 & -5.066641151 & -1.573687115 & -1.573687151 \\
\hline & 1 & -5.066291936 & -5.066292323 & -1.573677890 & -1.573677925 \\
\hline 2 & 0 & -5.007960488 & -5.007961116 & -1.568319272 & -1.568319330 \\
\hline & 1 & -5.007617702 & -5.007618329 & -1.568310094 & -1.568310152 \\
\hline & 2 & -5.006932272 & -5.006932904 & -1.568291737 & -1.568291796 \\
\hline & & & & & \\
\hline 3 & 0 & -4.950293764 & -4.950294624 & -1.568291737 & -1.562978927 \\
\hline & 1 & -4.949956880 & -4.949957740 & -1.562969715 & -1.562969796 \\
\hline & 2 & -4.949283254 & -4.949284119 & -1.562951452 & -1.562951533 \\
\hline & 3 & -4.948273160 & -4.948274034 & -1.562924059 & -1.562924140 \\
\hline & & & & & \\
\hline 4 & 0 & -4.893617382 & -4.893618469 & -1.557665652 & -1.557665755 \\
\hline & 1 & -4.893286268 & -4.893287355 & -1.557656567 & -1.557656670 \\
\hline & 2 & -4.892624178 & -4.892625268 & -1.557638398 & -1.557638501 \\
\hline & 3 & -4.891631378 & -4.891632476 & -1.557611144 & -1.557611248 \\
\hline & 4 & -4.890308272 & -4.890309388 & -1.557574807 & -1.557574911 \\
\hline & & & & & \\
\hline 5 & 0 & -4.837908798 & -4.837910103 & -1.552379504 & -1.552379630 \\
\hline & 1 & -4.837583322 & -4.837584627 & -1.552370466 & -1.552370591 \\
\hline & 2 & -4.836932504 & -4.836933812 & -1.552352389 & -1.552352514 \\
\hline & 3 & -4.835956606 & -4.835957923 & -1.552325274 & -1.552325399 \\
\hline & 4 & -4.834656026 & -4.834657357 & -1.552289121 & -1.552289247 \\
\hline & 5 & -4.833031292 & -4.833032637 & -1.552243934 & -1.552244060 \\
\hline & & & & & \\
\hline & & & & & \\
\hline
\end{tabular}

Our results are very consistent with the results obtained by Bayrak, Boztosun and Ciftci ${ }^{29}$. Also, it is observed that the energy eigenvalues become more bounded as the quantum states of these molecules increases. Moreover, with the introduction of the energy slope parameter, the energy eigenvalues for the different diatomic molecules tends to increase drastically (See Tables
4-6), as compared to the absence of energy slope parameter in Tables 2 and 3. 
Table 4. Energy eigenvalues (in eV) of energy dependent Kratzer molecular potential for different values of $n$ and $\ell$ for ground states $\mathrm{CO}, \mathrm{NO}, \mathrm{O}_{2}$ and $\mathrm{I}_{2}$ diatomic molecules, with $\eta=0.5$.

\begin{tabular}{|c|c|c|c|c|c|}
\hline$n$ & $\ell$ & $\mathrm{CO}$ & NO & $\mathrm{O}_{2}$ & $I_{2}$ \\
\hline 0 & 0 & -1.685470983 & -1.598084322 & -1.436597363 & -0.8821098304 \\
\hline \multirow[t]{2}{*}{1} & 0 & -1.679305517 & -1.590871796 & -1.427770406 & -0.8798579292 \\
\hline & 1 & -1.679233740 & -1.590790708 & -1.427673989 & -0.8798527834 \\
\hline \multirow[t]{3}{*}{2} & 0 & -1.673229781 & -1.583767938 & -1.419088479 & -0.8776168647 \\
\hline & 1 & -1.673159688 & -1.583688734 & -1.418994333 & -0.8776117488 \\
\hline & 2 & -1.673019611 & -1.583530439 & -1.418806171 & -0.8776015171 \\
\hline \multirow[t]{4}{*}{3} & 0 & -1.667239060 & -1.576767407 & -1.410544994 & -0.8753865376 \\
\hline & 1 & -1.667170554 & -1.576689982 & -1.410453001 & -0.8753814511 \\
\hline & 2 & -1.667033643 & -1.576535242 & -1.410269138 & -0.8753712785 \\
\hline & 3 & -1.666828530 & -1.576303400 & -1.409993648 & -0.8753560203 \\
\hline \multirow[t]{5}{*}{4} & 0 & -1.661329077 & -1.569865334 & -1.402133902 & -0.8731668498 \\
\hline & 1 & -1.661262070 & -1.569789595 & -1.402043954 & -0.8731617926 \\
\hline & 2 & -1.661128150 & -1.569638220 & -1.401864174 & -0.8731516783 \\
\hline & 3 & -1.660927510 & -1.569411413 & -1.401594794 & -0.8731365078 \\
\hline & 4 & -1.660660434 & -1.569109476 & -1.401236158 & -0.8731162818 \\
\hline \multirow[t]{6}{*}{5} & 0 & -1.655495943 & -1.563057265 & -1.393849636 & -0.8709577054 \\
\hline & 1 & -1.655430353 & -1.562983126 & -1.393761633 & -0.8709526770 \\
\hline & 2 & -1.655299265 & -1.562834945 & -1.393585738 & -0.8709426205 \\
\hline & 3 & -1.655102858 & -1.562612914 & -1.393322171 & -0.8709275367 \\
\hline & 4 & -1.654841404 & -1.562317323 & -1.392971259 & -0.8709074262 \\
\hline & 5 & -1.654515258 & -1.561948551 & -1.392533439 & -0.8708822905 \\
\hline
\end{tabular}


Table 5. Energy eigenvalues (in eV) of energy dependent Kratzer molecular potential for different values of $n$ and $\ell$ for ground states $\mathrm{CO}, \mathrm{NO}, \mathrm{O}_{2}$ and $\mathrm{I}_{2}$ diatomic molecules, with $\eta=1$.

\begin{tabular}{|c|c|c|c|c|c|}
\hline$n$ & $\ell$ & $\mathrm{CO}$ & NO & $\mathrm{O}_{2}$ & $I_{2}$ \\
\hline 0 & 0 & -0.9143283175 & -0.8879121698 & -0.8355872569 & -0.6120188036 \\
\hline \multirow[t]{2}{*}{1} & 0 & -0.9118740953 & -0.8849352196 & -0.8316829851 & -0.6107178506 \\
\hline & 1 & -0.9118356154 & -0.8848906520 & -0.8316274353 & -0.6107142849 \\
\hline \multirow[t]{3}{*}{2} & 0 & -0.9094616292 & -0.8820091632 & -0.8278482118 & -0.6094231767 \\
\hline & 1 & -0.9094242910 & -0.8819658826 & -0.8277942378 & -0.6094196340 \\
\hline & 2 & -0.9093497161 & -0.8818794293 & -0.8276864125 & -0.6094125490 \\
\hline \multirow[t]{4}{*}{3} & 0 & -0.9070878461 & -0.8791305048 & -0.8240785907 & -0.6081347096 \\
\hline & 1 & -0.9070515614 & -0.8790884168 & -0.8240260842 & -0.6081311896 \\
\hline & 2 & -0.9069790858 & -0.8790043406 & -0.8239211853 & -0.6081241500 \\
\hline & 3 & -0.9068706055 & -0.8788784754 & -0.8237641210 & -0.6081135914 \\
\hline \multirow[t]{5}{*}{4} & 0 & -0.9047500576 & -0.8762961642 & -0.8203702568 & -0.6068523785 \\
\hline & 1 & -0.9047147491 & -0.8762551852 & -0.8203191212 & -0.6068488809 \\
\hline & 2 & -0.9046442191 & -0.8761733203 & -0.8202169568 & -0.6068418862 \\
\hline & 3 & -0.9045386403 & -0.8760507547 & -0.8200639757 & -0.6068313948 \\
\hline & 4 & -0.9043982696 & -0.8758877638 & -0.8198604937 & -0.6068174077 \\
\hline \multirow[t]{6}{*}{5} & 0 & -0.9024458954 & -0.8735034094 & -0.8167197533 & -0.6055761139 \\
\hline & 1 & -0.9024114949 & -0.8734634655 & -0.8166699025 & -0.6055726385 \\
\hline & 2 & -0.9023427747 & -0.8733836647 & -0.8165703009 & -0.6055656880 \\
\hline & 3 & -0.9022398959 & -0.8732641797 & -0.8164211473 & -0.6055552630 \\
\hline & 4 & -0.9021030976 & -0.8731052679 & -0.8162227378 & -0.6055413644 \\
\hline & 5 & -0.9019326946 & -0.8729072681 & -0.8159754631 & -0.6055239934 \\
\hline
\end{tabular}


Table 6. Energy eigenvalues (in eV) of energy dependent Kratzer molecular potential for different values of $n$ and $\ell$ for ground states $\mathrm{CO}, \mathrm{NO}, \mathrm{O}_{2}$ and $\mathrm{I}_{2}$ diatomic molecules, with $\eta=2$.

\begin{tabular}{|c|c|c|c|c|c|}
\hline$n$ & $\ell$ & $\mathrm{CO}$ & NO & $\mathrm{O}_{2}$ & $I_{2}$ \\
\hline 0 & 0 & -0.4774929881 & -0.4701556293 & -0.4550063750 & -0.3795921052 \\
\hline \multirow[t]{2}{*}{1} & 0 & -0.4765718304 & -0.4690143732 & -0.4534445388 & -0.3789570351 \\
\hline & 1 & -0.4765520739 & -0.4689911448 & -0.4534147167 & -0.3789548282 \\
\hline \multirow[t]{3}{*}{2} & 0 & -0.4756699234 & -0.4678965624 & -0.4519148098 & -0.3783251918 \\
\hline & 1 & -0.4756509229 & -0.4678741908 & -0.4518860464 & -0.3783230016 \\
\hline & 2 & -0.4756130152 & -0.4678295473 & -0.4518286336 & -0.3783186218 \\
\hline \multirow[t]{4}{*}{3} & 0 & -0.4747853199 & -0.4667999700 & -0.4504144000 & -0.3776965243 \\
\hline & 1 & -0.4747669984 & -0.4667783719 & -0.4503865989 & -0.3776943507 \\
\hline & 2 & -0.4747304393 & -0.4667352661 & -0.4503311006 & -0.3776900040 \\
\hline & 3 & -0.4746758092 & -0.4666708312 & -0.4502481107 & -0.3776834845 \\
\hline \multirow[t]{5}{*}{4} & 0 & -0.4739163936 & -0.4657227183 & -0.4489409312 & -0.3770709835 \\
\hline & 1 & -0.4738986862 & -0.4657018233 & -0.4489140099 & -0.3770688262 \\
\hline & 2 & -0.4738633478 & -0.4656601156 & -0.4488602625 & -0.3770645119 \\
\hline & 3 & -0.4738105292 & -0.4655977580 & -0.4487798774 & -0.3770580413 \\
\hline & 4 & -0.4737404532 & -0.4655149913 & -0.4486731336 & -0.3770494152 \\
\hline \multirow[t]{6}{*}{5} & 0 & -0.4730617690 & -0.4646632066 & -0.4474923542 & -0.3764485216 \\
\hline & 1 & -0.4730446207 & -0.4646429544 & -0.4474662416 & -0.3764463803 \\
\hline & 2 & -0.4730103939 & -0.4646025252 & -0.4474141038 & -0.3764420979 \\
\hline & 3 & -0.4729592263 & -0.4645420684 & -0.4473361143 & -0.3764356752 \\
\hline & 4 & -0.4728913214 & -0.4644618046 & -0.4472325300 & -0.3764271130 \\
\hline & 5 & -0.4728069445 & -0.4643620221 & -0.4471036873 & -0.3764164124 \\
\hline
\end{tabular}

But, the variation of the quantum state with the energy eigenvalues in the presence of the energy slope parameter is in the reverse direction. As the quantum number increases, the energy eigenvalues of energy dependent molecular Kratzer potential becomes more bounded.
Figures 2 and 3 shows the energy eigenvalues variation with the energy slope parameter for different quantum numbers. Both graphs show a similar close trend of increase in energy eigenvalues as the energy slope parameter increases, for different quantum numbers. 


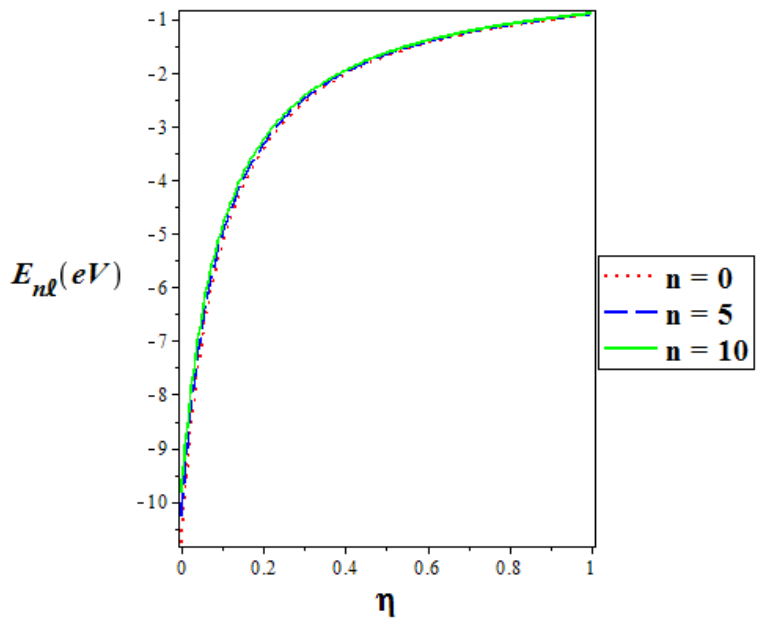

Figure 2. Energy eigenvalues of energy-dependent Kratzer molecular potential vs $\eta$ for different values of $n$.

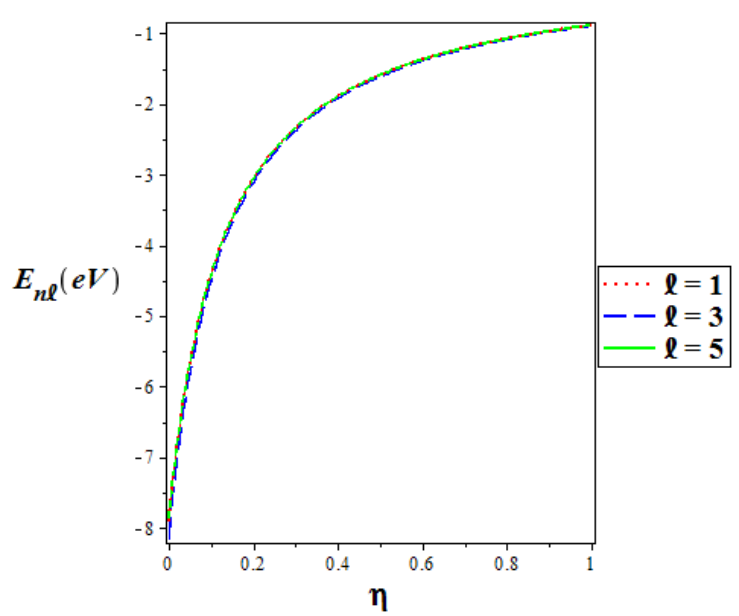

Figure 3. Energy eigenvalues of energy-dependent Kratzer Molecular Potential Vs $\eta$ for different values of $\ell$.

In Fig. 4, there is a diverse behavior of the energy eigenvalues with the potential strength, for different values of the energy slope parameter. Here, there is a direct decrease in energy eigenvalue as the potential strength increase in the absence of the energy slope parameter $(\eta=0)$. With the presence of the energy slope parameter $(\eta=-1)$, the energy eigenvalue becomes very bounded with a maximum potential strength of about $1 \mathrm{eV}$. Similarly, there is a stunted decrease in energy eigenvalue (not more than $1 \mathrm{eV}$ ), as the potential strength increase $(\eta=1)$.

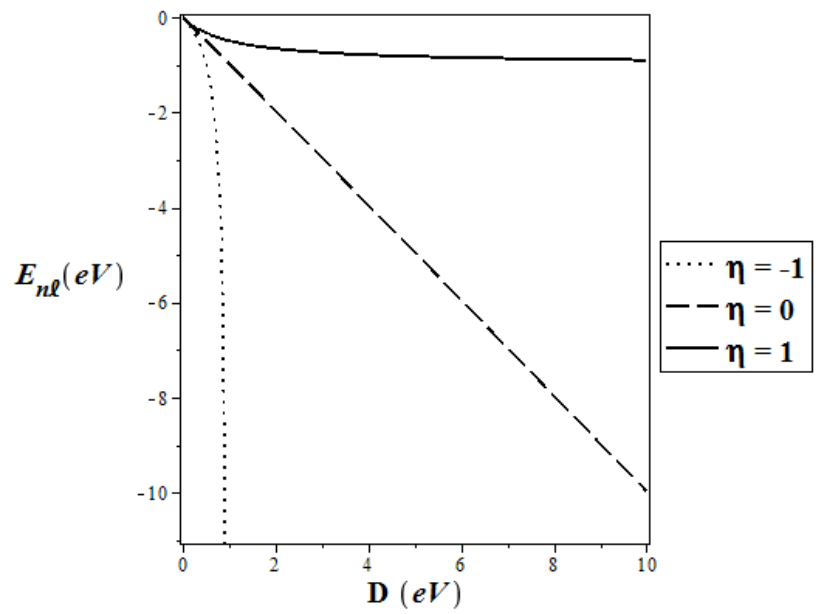

Figure 4. Energy eigenvalues of energy-dependent Kratzer molecular potential vs $D$ for different values of $\eta$.

\section{Conclusions}

We applied the asymptotic iteration method (AIM) to solve the Schrödinger equation with energy dependent molecular Kratzer potential. Its energy eigenvalues and corresponding wave functions in terms of confluent hypergeometric function have been obtained. The numerical results of the energy eigenvalues have been presented in the presence and absence of the energy slope parameter, respectively, for four different diatomic molecules $\left(\mathrm{CO}, \mathrm{NO}, \mathrm{O}_{2}\right.$ and $\mathrm{I}_{2}$ ). Our results agree with the results in available literature, especially when the energy slope parameter is set to zero. We have also shown graphically the variation of the energy eigenvalues with some of the potential parameters like energy slope parameter and the potential strength. The behavior of the energy eigenvalues with these parameters is similar in all the diatomic molecules studied. The result obtained in this study finds application in quantum chemistry, molecular physics amongst others.

\section{Acknowledgment}

The authors thank the kind reviewers for the positive comments and suggestions that lead to an improvement of our manuscript

\section{References}

[1] Chun-Feng, H., Zhong-Xiang, Z., Yan, L., Bound states of the Klein-Gordon equation with vector and scalar Wood-Saxon potentials, Acta Physica Sinica 8 (8) 
(1999) 561-565. https://doi.org/10.1088/1004$423 X / 8 / 8 / 001$.

[2] Sever, R., Tezan, C., Yesiltas, O., Bucurgat, M., Exact Solution of Effective Mass Schrödinger Equation for the Hulthen Potential, International Journal of Theoretical Physics 47 (9) (2008) 2243-2248. https://doi.org/10.1007/s10773-008-9656-7.

[3] Ikhdair, S. M., An approximate $\kappa$ state solutions of the Dirac equation for the generalized Morse potential under spin and pseudospin symmetry, Journal of Mathematical Physics 52 (5) (2011) 1-22. https://doi.org/10.1063/1.3583553.

[4] Maghsoodi, E., Hassanabadi, H., Zarrinkamar, S., Spectrum of Dirac Equation Under Deng-Fan Scalar and Vector Potentials and a Coulomb Tensor Interaction by SUSYQM, Few-Body Systems 53 (3-4) (2012) 525538. https://doi.org/10.1007/s00601-012-0314-5.

[5] Ikot, A. N., Akpabio, L. E., Umoren, E. B., Exact Solution of Schrödinger Equation with Inverted WoodsSaxon and Manning-Rosen Potential, Journal of Scientific Research 3 (1) (2011) 25-33. https://doi.org/10.3329/jsr.v3i1.5310.

[6] Qiang, W. C., Dong, S. H., Analytical approximations to the solutions of the Manning-Rosen potential with centrifugal term, Physics Letters A 368 (1-2) (2007) 13-17. https://doi.org/10.1016/j.physleta.2007.03.057.

[7] Yahya, W. A., Oyewumi, K. J., Thermodynamic properties and approximate solutions of the $\ell$-state Pöschl-Teller-type potential, Journal of the Association of Arab Universities for Basic and Applied Sciences 21 (1) (2016) 53-58. https://doi.org/10.1016/j.jaubas.2015.04.001.

[8] Sun, Y., He, S., Jia, C-S., Equivalence of the deformed modified Rosen-Morse potential energy model and the Tietz potential energy model, Physica Scripta 87 (2) (2013) 1-5. https://doi.org/10.1088/00318949/87/02/025301.

[9] Edet, C. O., Okoi P. O., Any 1-State Solutions of the Schrödinger Equation for q Deformed Hulthen plus Generalized Inverse Quadratic Yukawa Potential in Arbitrary Dimensions, Revista Mexicana de Física 65 (2019) 333-344.

https://doi.org/10.31349/RevMexFis.65.333.

[10] Edet, C. O., Okorie, U. S., Ngiangia, A. T., Ikot, A. N., Bound state solutions of the Schrödinger equation for the modified Kratzer potential plus screened Coulomb potential, Indian Journal of Physics
(2019) 1-9. https://doi.org/10.1007/s12648-019-014779.

[11] Edet, C. O., Okoi P. O., Chima S. O, Analytic solutions of the Schrödinger equation with non-central generalized inverse quadratic Yukawa potential, Revista Brasileira de Ensino de Física 42 (e20190083) (2019) 19. https://doi.org/10.1590/1806-9126-RBEF-20190083.

[12] Ciftci, H., Hall, R. L., Saad, N., Asymptotic iteration method for eigenvalue problems, Journal of Physics A: Mathematical and General 36 (47) (2003) $11807-11816$. https://doi.org//10.1088/0305$4470 / 36 / 47 / 008$

[13] Falaye, B. J., Any $\ell$-state solutions of the Eckart potential via asymptotic iteration method, Central European Journal of Physics 10 (4) (2012) 960-965. https://doi.org/10.2478/s11534-012-0047-6.

[14] Qiang, W. C., Dong, S. H., Proper quantization rule, EPL Europhysics Letters 89 (1) (2010) 10003.110003.2. https://doi.org/10.1209/0295-5075/89/10003.

[15] Ikhdair, S. M., Sever, R., Exact quantization rule to the Kratzer-type potentials: an application to the diatomic molecules, Journal of Mathematical Chemistry $45 \quad$ (4) (2009) 1137-1152. https://doi.org/10.1007/s10910-008-9438-8.

[16] Okorie, U. S., Ikot, A. N., Edet, C. O., Akpan, I. O., Sever, R., Rampho, R., Solutions of the Klein Gordon equation with generalized hyperbolic potential in Ddimensions, Journal of Physics Communication 3 (095015) (2019). https://doi.org/10.1088/2399$6528 / \mathrm{ab} 42 \mathrm{c} 6$

[17] Onate, C. A., Ojonubah, J. O., Eigensolutions of the Schrödinger equation with a class of Yukawa potentials via supersymmetric approach, Journal of Theoretical and Applied Physics 10 (1) (2016) 21-26. https://doi.org/10.1007/s40094-015-0196-2.

[18] Ikot, A. N., Obong, H. P., Abbey, T. M., Zare, S., Ghafourian, M., Hassanabadi, H., Bound and scattering state of position dependent mass Klein-Gordon equation with Hulthen plus deformed-type hyperbolic potential, Few-Body Systems 57 (9) (2016) 807-822. https://doi.org/10.1007/s00601-016-1111-3.

[19] Onate, C. A., Onyeaju, M. C., Ikot, A. N., Ojonubah, J. O., Analytical solutions of the KleinGordon equation with a combined potential, Chinese Journal of Physics 54 (5) (2016) 820-829. https://doi.org/10.1016/j.cjph.2016.08.007. 
[20] Onate, C. A., Ikot, A. N., Onyeaju, M. C., Udoh, M. E., Bound state solutions of D-dimensional KleinGordon equation with hyperbolic potential, Karbala International Journal of Modern Science 3 (1) (2017) 17. https://doi.org/10.1016/j.kijoms.2016.12.001.

[21] Sadeghi, J., Factorization method and solution of the non-central modified Kratzer potential, Acta Physica $\begin{array}{lllll}\text { Polonica A } & 112 \quad \text { (1) 23-28. }\end{array}$ http://inspirehep.net/record/1426929/files/a112z103.pd f.

[22] Kratzer, A., Die ultraroten rotationsspektren der halogenwasserstoffe, Zeitschrift für Physik A Hadrons and Nuclei $3 \quad$ (5) (1920) 289-307. https://doi.org/10.1007/BF01327754.

[23] LeRoy, R. J., Bernstein, R. B., Dissociation energy and long-range potential of diatomic molecules from vibrational spacings of higher levels, The Journal of Chemical Physics 52 (8) (1970) 3869-3879. https://doi.org/10.1063/1.1673585.

[24] Ikhdair, S. M., An approximate $\kappa$ state solutions of the Dirac equation for the generalized Morse potential under spin and pseudospin symmetry, Journal of Mathematical Physics 52 (5) (2011) 052303.1052303.22. https://doi.org/10.1063/1.3583553.

[25] Saad, N., Hall, R. J., Cifti H., The Klein-Gordon equation with the Kratzer potential in d dimensions, Central European Journal of Physics 6 (3) (2008) 717729. https://doi.org/10.2478/s11534-008-0022-4.

[26] Hassanabadi, H., Rahimov, H., Zarrinkamar, S., Approximate solutions of Klein-Gordon equation with Kratzer potential, Advances in High Energy Physics 2011 (458087). https://doi.org/10.1155/2011/458087.

[27] Budaca R., Bohr Hamiltonian with an energydependent $\gamma$-unstable Coulomb-like potential, The European Physical Journal A 52 (2016) 314. https://doi.org/10.1140/epja/i2016-16314-8.

[28] Lombard, R. J., Mareš. J., The many-body problem with an energy-dependent confining potential, Physics Letters A $373 \quad$ (4) (2009) 426-429. https://doi.org/10.1016/j.physleta.2008.12.009.

[29] Bayrak, O., Boztosun, I., Ciftci, H., Exact analytical solutions to the kratzer potential by the asymptotic iteration method, International Journal of Quantum $\begin{array}{lllll}\text { Chemistry } & 107 & \text { (3) } & \text { (2007) 540-544. }\end{array}$ https://doi.org/10.1002/qua.21141.

[30] Hassanabadi, H., Zarrinkamar, S., Rajabi, A. A., Exact solutions of D-dimensional Schrödinger equation for an energy-dependent potential by NU method, Communications in Theoretical Physics 55 (4) (2011) 541-544. https://doi.org/10.1088/0253-6102/55/4/01.

[31] Hassanabadi, H., Zarrinkamar, S., Hamzavi, H., Rajabi, A. A., Exact solutions of D-dimensional KleinGordon equation with an energy-dependent potential by using of Nikiforov-Uvarov method, Arabian Journal for Science and Engineering 37 (1) (2012) 209-215. https://doi.org/10.1007/s13369-011-0168-z.

[32] Boumali, A., Labidi, M., Shannon entropy and Fisher information of the one-dimensional KleinGordon oscillator with energy-dependent potential, Modern Physics Letters A 33 (6) (2018) 1-26. https://doi.org/10.1142/S0217732318500335.

[33] Lombard, R. J., Mareš, J., Volpe, C., Wave equation with energy-dependent potentials for confined systems, Journal of Physics G: Nuclear and Particle

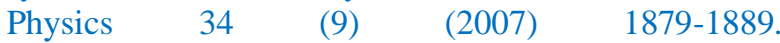
https://doi.org/10.1088/0954-3899/34/9/002.

[34] Gupta, P., Mehrotra, I., Study of heavy quarkonium with energy dependent Potential, Journal of Modern

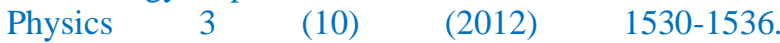
https://doi.org/10.4236/jmp.2012.310189.

[35] Ikot, A. N., Chukwuocha, E. O., Onyeaju, M. C., Onate, C. A., Ita, B. I., Udoh, M. E., Thermodynamics properties of diatomic molecules with general molecular potential, Pramana $90 \quad$ (2) (2018) 22-30. https://doi.org/10.1007/s12043-017-1510-0.

[36] Falaye, B. J., Corrigendum: Energy spectrum for trigonometric Pöschl-Teller potential solved by the asymptotic iteration method, Canadian Journal of $\begin{array}{llllll}\text { Physics } & 91 & \text { (4) 365-367. }\end{array}$ https://doi.org/10.1139/cjp-2013-0011. 